\title{
PAPER
}

\section{Magnetic resonance brain imaging in people with dizziness: a comparison with non-dizzy people}

\author{
N Colledge, S Lewis, G Mead, R Sellar, J Wardlaw, J Wilson
}

J Neurol Neurosurg Psychiatry 2002;72:587-589

See end of article for authors' affiliations

.....................

Correspondence to:

Dr Gillian Mead,

Department of Clinical and

Surgical Sciences (Geriatric

Medicine), 21 Chalmers

Street, Edinburgh

EH3 9EW, UK:

gillian.e.mead@ed.ac.uk

Received 2 August 2001

In revised form

8 January 2002

Accepted

10 January 2002

\begin{abstract}
Background: Dizziness is a common symptom affecting about $30 \%$ of people over the age of 65 .
Objective: To investigate the hypothesis that structural abnormalities of the brain and cervical cord are more common in dizzy than in non-dizzy subjects.

Methods: A case-control study of subjects over the age of 65 with and without dizziness: 125 dizzy subjects and 86 non-dizzy subjects were recruited from the community through articles in the local press. Magnetic resonance imaging (MRI) of the brain and neck was performed, and was read by a consultant neuroradiologist blind to the clinical details.

Results: All dizzy subjects and all controls had at least one structural abnormality. Cerebral atrophy was found in $86 \%$ of dizzy subjects and $85 \%$ controls ( $p=1.0$ ) At least one white matter lesion was found in $69 \%$ of dizzy subjects and $78 \%$ controls $(p=0.21)$. White matter lesions in the midbrain were more common in dizzy than in non-dizzy subjects $(22 \% \vee 4 \%, p<0.001)$. There were no significant differences in the prevalence of cord compression, cervical subluxation, facet joint degeneration, vertebral artery compression, or vertebral artery occlusion between dizzy and non-dizzy subjects.

Conclusions: Structural abnormalities of the brain and neck are common in both dizzy and non-dizzy subjects. "Routine" MRI is unlikely to reveal a specific cause for dizziness. The observation of more frequent white matter lesions in the midbrain in dizzy subjects requires further study to determine whether small vessel changes could cause dizziness in older people.
\end{abstract}

$\mathrm{D}$ izziness is a common symptom which affects about $30 \%$ of people over the age of $65 .{ }^{12}$ Older people often find it difficult to articulate the nature of their symptoms, but "dizziness" includes sensations such as giddiness, faintness, "light headedness", vertigo, and imbalance. It is associated with depressive symptoms, poor self rated health, falls, and a reduction in social activities. ${ }^{3}$ We have previously shown that the most common clinical causes of dizziness in older people in the community are central vascular disease (defined as unsteadiness with or without light headedness in association with an abnormal gait, marche a petits pas) ${ }^{4}$ or cervical spondylosis (defined as symptoms of dizziness on head or neck movement with reduced range of neck movements). ${ }^{5}$ Thus it is reasonable to suggest that dizziness may be caused by structural problems in the brain or cervical spine. For example, a feeling of unsteadiness may be related to disease of the cerebellum and its connections; vertigo may be related to disease of the cerebellum, its connections, or the VIII nerve; and light headedness may be caused by changes in cerebral blood flow, which may over a long period of time induce structural abnormalities such as white matter lesions.

The presence of structural abnormalities on brain imaging in people with dizziness has not been widely studied. In a study of 79 patients aged between 19 and 59 with rotatory vertigo or "some other manifestation of dizziness" it was found that $34 \%$ had at least one abnormality on computed tomography or magnetic resonance brain imaging which was thought to be relevant to the aetiology of dizziness-for example, atrophy, infarction, or demyelination. ${ }^{6}$ In another study of 21 elderly patients with dizziness, ischaemic changes on magnetic resonance imaging (MRI) were present in six of the eight patients who underwent imaging. ${ }^{7}$ Findings such as these have led to the suggestion that brain imaging might be useful in elucidating the cause of dizziness. ${ }^{6}$ However, such abnormalities on structural brain imaging are also common in people without dizziness, ${ }^{89}$ and a small study of 20 elderly subjects with dizziness and nine nondizzy subjects found no difference in the prevalence of white matter lesions between the two groups (although the lack of association may simply reflect the small numbers of people recruited to the study). ${ }^{10}$ There is, however, some evidence that structural brain lesions (for example cerebral atrophy and white matter lesions) are more common in people who complain of disequilibrium, poor balance, and falls (as opposed to the rather more non-specific complaint of "dizziness") than in those without these symptoms. ${ }^{112}$

We have briefly reported preliminary results from MRI of the brain and cervical cord in the dizzy and non-dizzy subjects recruited to our community based study. We now report our radiological findings in full.

\section{METHODS}

Subjects over the age of 65 were invited to take part in the study through articles in the local press and through our local survey of dizziness. ${ }^{2}$ We obtained signed consent and permission from each person's general practitioner before formal recruitment. Only those who had suffered from dizziness for three months or more were recruited to the dizzy group, and only those who had never been dizzy were recruited to the control group. No other inclusion or exclusion criteria were applied, and the dizzy subjects were not matched for age and other clinical characteristics with the non-dizzy subjects. The definition of dizziness included unsteadiness, vertigo or "light headedness", or a combination of these symptoms.

We recorded dizzy symptoms, medical history, current treatments, and functional ability. One of us (NC) performed all the clinical assessments, which included visual acuity testing. Posturography and vestibular testing were also performed, and these results were reported in a previous paper. ${ }^{5}$ The assessments were done blind to the MRI.

MRI of the head and neck was done with a Siemens 1.5 Tesla scanner. Subjects with cardiac pacemakers, intraocular metallic foreign bodies, or intracranial ferromagnetic clips did not undergo scanning. Images were reported according to a 
standardised format by a consultant neuroradiologist (RJS) who was blind to whether or not the scans were of a dizzy or non-dizzy subject. Cerebral atrophy was categorised subjectively as mild, moderate, or severe; white matter lesions were defined as an abnormal signal less than $3 \mathrm{~mm}$ in diameter (not perivascular); and cerebral infarcts were areas of high signal intensity on $\mathrm{T} 2$ images, typically involving the grey and white matter without mass effect. Cord compression was categorised subjectively as mild or severe; subluxation as none, $0-1 \mathrm{~cm}$, or more than $1 \mathrm{~cm}$; facet joint degeneration subjectively as none, mild, moderate, or severe; and vertebral artery compression subjectively as mild, moderate, or severe. Vertebral artery occlusion was identified by a flow void in the appropriate place.

\section{Statistics}

Statistical analysis was performed using the Statistical Package for the Social Sciences (SPSS). We used $\chi^{2}$ tests, Fisher's exact test, and the Mantel-Haenszel test as appropriate to compare MRI abnormalities in the dizzy and non-dizzy subjects.

\section{RESULTS}

We recruited 149 subjects with dizziness and 97 controls. The mean (SD) age of the dizzy subjects was 76 (6) years and 69 $(49 \%)$ were men. The mean age of the controls was 76 (5) years and 39 (40\%) were men. A history of smoking, ischaemic heart disease, stroke, ear disease, and eye disease was significantly more common in dizzy subjects than the controls. ${ }^{5}$ Dizzy subjects were more likely to be taking diuretics, calcium antagonists, and aspirin. ${ }^{5}$ One hundred and sixteen dizzy subjects $(77 \%)$ described their symptoms as unsteadiness, 89 $(60 \%)$ as light headedness, and $37(32 \%)$ as vertigo, while 83 $(56 \%)$ described more than one sensation. Based on standard diagnostic criteria, the most common diagnoses in the dizzy group were central vascular disease $(105 ; 70 \%),{ }^{4}$ cervical spondylosis $(98 ; 66 \%)$, and anxiety or hyperventilation (48; $32 \%) .^{5}$ There was considerable overlap of symptoms, with 126 $(85 \%)$ having more than one diagnosis. ${ }^{5}$

One hundred and twenty five dizzy subjects and 86 non-dizzy subjects underwent MRI. In the remainder, scanning was contraindicated, refused, or not tolerated.

The main findings were as follows. All dizzy subjects and all controls had at least one structural abnormality. Some degree of cerebral atrophy was found in more than three quarters of dizzy and non-dizzy subjects (table 1). Most subjects, both dizzy and non-dizzy, had at least one white matter lesion (table 2) and the proportions with more extensive hemispheric white matter lesions were very similar in the two groups. Midbrain white matter lesions (but not pons or brain stem lesions) were significantly more common in dizzy subjects (table 2). There was no significant difference in the prevalence of disease of the cervical cord and vertebrae in the dizzy and non-dizzy subjects (table 3 ), or in the presence of disease of the VIII nerve and semicircular canals (table 4).

\section{DISCUSSION}

To our knowledge, this is the largest case-control study of dizzy and non-dizzy people to undergo MRI of their brain and neck. We found no significant differences in the prevalence of cerebral atrophy, the number of white matter lesions, the number of cerebral infarcts, or disease of the semicircular canals or cerebellopontine angle in subjects with and without dizziness, though dizzy subjects were more likely to have cardiovascular risk factors and a history of previous stroke. This suggests that routine MRI in patients with dizziness is unlikely to be helpful, and does not support the view that "head CT and MRI are diagnostically useful when dizziness of a CNS aetiology is suspected," as previously suggested. ${ }^{6}$ In older people, dizziness is often attributed to vertebrobasilar ischaemia, which is assumed to be caused by
Table 1 Cerebral hemispheres in dizzy and non-dizzy subjects

\begin{tabular}{|c|c|c|c|}
\hline & $\begin{array}{l}\text { Dizzy } \\
\text { subjects } \\
\text { ( } n=125)\end{array}$ & $\begin{array}{l}\text { Non-dizzy } \\
\text { subjects } \\
(n=86)\end{array}$ & $\mathrm{p}$ Value \\
\hline Atrophy & & & $0.47^{*}$ \\
\hline None & $17(14)$ & $12(15)$ & \\
\hline Mild & $38(31)$ & $28(34)$ & \\
\hline Moderate & $51(42)$ & $35(43)$ & \\
\hline Severe & $16(13)$ & $7(8)$ & \\
\hline$\ddagger$ No information & 3 & 4 & \\
\hline White matter lesion & & & $0.21 \dagger$ \\
\hline 0 & $38(31)$ & $18(22)$ & \\
\hline $1-5$ & $40(33)$ & $32(39)$ & \\
\hline $6-10$ & $21(17)$ & $15(18)$ & \\
\hline$>10$ & $24(20)$ & $17(21)$ & \\
\hline$\ddagger$ No information & 2 & 4 & \\
\hline Infarct & & & $0.70^{*}$ \\
\hline None & $118(96)$ & $80(98)$ & \\
\hline Occipital & $0(0)$ & $2(2)$ & \\
\hline Parietal & $0(0)$ & $0(0)$ & \\
\hline $\begin{array}{l}\text { Middle cerebral artery } \\
\text { territory }\end{array}$ & $1(1)$ & $0(0)$ & \\
\hline Lacunar & $3(2)$ & $0(0)$ & \\
\hline Occipital and parietal & $1(1)$ & $0(0)$ & \\
\hline$\ddagger$ No information & 2 & 4 & \\
\hline
\end{tabular}

Values are $\mathrm{n}(\%)$. The denominator used to calculate the percentage is the number of scans where information is available.

*Manzel-Haenszel test for linear association; $\dagger \chi^{2}$ test or Fisher's exact test as appropriate (some abnormality $v$ no abnormality).

$\ddagger$ Scans unclear or unreadable.

Table 2 Site of posterior fossa white matter lesions in dizzy and non-dizzy subjects

\begin{tabular}{|c|c|c|c|}
\hline & $\begin{array}{l}\text { Dizzy } \\
\text { subjects } \\
(n=125)\end{array}$ & $\begin{array}{l}\text { Non-dizzy } \\
\text { subjects } \\
(n=86)\end{array}$ & $\begin{array}{l}\mathrm{p} \text { Value } \\
\left(\chi^{2} \text { test }\right)\end{array}$ \\
\hline Lesion in pons & $16(13)$ & $10(13)$ & 1.00 \\
\hline No lesion in pons & $103(87)$ & 69 (87) & \\
\hline Lesion in midbrain & $26(22)$ & $3(4)$ & $<0.001$ \\
\hline No lesion in midbrain & 93 (78) & $76(96)$ & \\
\hline Lesion in medulla & $8(7)$ & $5(6)$ & 1.00 \\
\hline No lesion in medulla & $111(93)$ & $74(94)$ & \\
\hline *No information & 6 & 7 & \\
\hline
\end{tabular}

Values are $n(\%)$. The denominator used to calculate the percentage is the number of scans where information is available.

* Scans unclear or unreadable.

impaired blood flow through the vertebral arteries as a result of cervical spine disease. We found no difference in disease of the cervical cord and vertebrae in dizzy and non-dizzy subjects. Hence a diagnosis of 'vertebrobasilar ischaemia' in dizzy people is probably unhelpful, and the application of such a label may prevent physicians from considering other potential causes of dizziness.

The only difference in MRI between dizzy and non-dizzy subjects was that midbrain white matter lesions were more common in the dizzy subjects. There are several possible explanations for this finding. First, it may be a chance finding, but the $p$ value of $<0.001$ makes this highly unlikely. Second, it may be related to the higher prevalence of cardiovascular risk factors in the dizzy group, but if this were the case, one might expect dizzy subjects to have more white matter lesions in all sites (and not just in the midbrain). Third, it is possible that these lesions cause dizziness in some patients. Midbrain lesions such as infarcts typically cause eye movement problems. ${ }^{13}$ Although our subjects did not have eye movement 
Table 3 Results of magnetic resonance imaging of the cervical cord and vertebrae in dizzy and non-dizzy subjects

\begin{tabular}{|c|c|c|c|}
\hline & $\begin{array}{l}\text { Dizzy } \\
\text { group } \\
(n=125)\end{array}$ & $\begin{array}{l}\text { Non-dizzy } \\
\text { group } \\
(n=86)\end{array}$ & $\mathrm{p}$ Value \\
\hline Cord compression & & & 0.81 * \\
\hline None & $53(44)$ & $38(45)$ & \\
\hline Mild & $58(49)$ & $38(45)$ & \\
\hline Severe & $8(7)$ & $8(10)$ & \\
\hline$\ddagger$ No information & 6 & 2 & \\
\hline Subluxation & & & $0.51 \dagger$ \\
\hline None & $113(95)$ & 77 (92) & \\
\hline $0-1 \mathrm{~cm}$ & $6(5)$ & $7(8)$ & \\
\hline$>1 \mathrm{~cm}$ & 0 (0) & $0(0)$ & \\
\hline$\ddagger$ No information & 6 & 2 & \\
\hline Facet joint degeneration & & & $0.16^{*}$ \\
\hline None & $32(27)$ & $27(32)$ & \\
\hline Mild & $31(26)$ & $25(30)$ & \\
\hline Moderate & $32(27)$ & $21(25)$ & \\
\hline Severe & $24(20)$ & $11(13)$ & \\
\hline$\ddagger$ No information & 6 & 2 & \\
\hline Vertebral artery compression & & & $0.16 \dagger$ \\
\hline None & $63(53)$ & $53(64)$ & \\
\hline Right & $15(13)$ & $7(8)$ & \\
\hline Left & $14(12)$ & $7(8)$ & \\
\hline Bilateral & $27(23)$ & 16 (19) & \\
\hline$\ddagger$ No information & 6 & 3 & \\
\hline Vertebral artery occlusion & & & $1.0 \dagger$ \\
\hline None & $90(76)$ & $63(76)$ & \\
\hline Right & $17(14)$ & $6(7)$ & \\
\hline Left & $8(7)$ & $12(15)$ & \\
\hline Bilateral & $4(3)$ & $2(2)$ & \\
\hline$\ddagger$ No information & 6 & 3 & \\
\hline
\end{tabular}

Values are $\mathrm{n}(\%)$. The denominator used to calculate the percentage is the number of scans where information is available.

*Mantel-Haenszel test for linear association; $\dagger \chi^{2}$ square test (some abnormality $v$ no abnormality).

$\ddagger$ Scans unclear or unreadable.

Table 4 Abnormalities of the VIII nerve and semicircular canals

\begin{tabular}{|c|c|c|}
\hline & $\begin{array}{l}\text { Dizzy } \\
\text { subjects } \\
(n=125)\end{array}$ & $\begin{array}{l}\text { Non-dizzy } \\
\text { subjects } \\
(n=86)\end{array}$ \\
\hline \multicolumn{3}{|l|}{ Acoustic neuroma } \\
\hline Yes & $0(0)$ & $0(0)$ \\
\hline No & $123(100)$ & $82(100)$ \\
\hline *No information & 2 & 4 \\
\hline \multicolumn{3}{|l|}{ Other } \\
\hline None & $118(96)$ & $79(96)$ \\
\hline Cerebellar atrophy & $1(1)$ & $0(0)$ \\
\hline Abnormal mastoid & $3(2)$ & $2(2)$ \\
\hline Vascular loop around L VII and VIII & $0(0)$ & $1(1)$ \\
\hline High jugular bulb behind IAM & $1(1)$ & $0(0)$ \\
\hline *No information & 2 & 4 \\
\hline
\end{tabular}

Frequencies are too low in some cells for valid statistical tests. IAM, internal auditory meatus.

problems, it is plausible that the clinical effect of infarcts may be different from insidious white matter lesions. ${ }^{13}$ Hence the clinical significance of these midbrain lesions remains uncertain.

We identified our subjects and controls from articles in the local press. Hence, our subjects may have had milder dizziness than people who seek medical attention for this symptom. Had we performed MRI in patients who present to their doctor with dizziness or who were admitted to hospital with dizziness-that is, the more severe end of the dizzy spectrum-we may have found a higher prevalence of structural brain abnormalities in the dizzy subjects, although one would not necessarily be able to attribute a causative role to such abnormalities. Had we just recruited patients with poor balance or falls, as in two previous studies, ${ }^{11}{ }^{12}$ we might have found a higher prevalence of structural abnormalities. However, we were interested in the complaint of dizziness in general rather than in more specific symptoms such as vertigo. Over half the patients with dizziness complained of more than one symptom, which meant that it was not possible to compare the MRI findings in patients with unsteadiness, vertigo, or light headedness.

\section{Implications}

These data suggest that routine MRI in the investigation of dizziness is unhelpful, because most abnormalities (for example, cerebral atrophy and white matter lesions) are equally common in people with and without dizziness. Obviously, if a specific diagnosis such as an acoustic neuroma is suspected, then MRI should be performed. The observation of more frequent white matter lesions in the midbrain in dizzy subjects requires further study to determine whether small vessel changes could cause dizziness in older people. Studies to determine the relation between falls, poor balance, and structural brain lesions would be interesting.

\section{ACKNOWLEDGEMENTS}

This study was funded by Research into Ageing (ref 9/110) and the Chief Scientist's Office, Scottish Office Home and Health Department (ref K/MRS/50/C1739).

\section{Authors' affiliations}

N Colledge, Department of Geriatric Medicine, Liberton Hospital and Royal Infirmary, Edinburgh, UK

S Lewis, G Mead, Department of Clinical and Surgical Sciences, (Geriatric Medicine) University of Edinburgh

R Sellar, Department of Neuroradiology, Western General Hospital, Edinburgh

J Wardlaw, Department of Neuroradiology, University of Edinburgh J Wilson, Department of Head and Neck Surgery, University of Newcastle, Newcastle upon Tyne, UK

\section{REFERENCES}

1 Sloane P, Blazer D, George LK. Dizziness in a community of elderly population. J Am Geriatr Soc 1989;37:101-8.

2 Colledge NR, Wilson JA, Macintyre CCA, et al. The prevalence of dizziness in an elderly community. Age Ageing 1994;23:117-20.

3 Tinetti ME, Williams CS, Gill TM. Health, functional and psychological outcomes among older persons with chronic dizziness. J Am Geriatr Soc 2000;48:417-21

4 Hopkins A. Clinical neurology. A modern approach. Oxford: Oxford University Press, 1993.

5 Colledge NR, Barr-Hamilton RM, Lewis SJ, et al. Evaluation of investigations to diagnose the cause of dizziness in elderly people: a community based controlled study. BM 1996;313:788-92

6 Ojala M, Ketonen L, Palo J. The value of CT and very low field MRI in the etiological diagnosis of dizziness. Acta Neurol Scand 1988:78:26-9.

7 Ahmad N, Wilson JA, Barr-Hamilton RM, et al. The evaluation of dizziness in elderly people. Postgrad Med J 1992;68:558-61.

8 Manolio TA, Kronmal RA, Burke GL, et al. Magnetic resonance abnormalities and cardiovascular disease in older adults. The Cardiovascular Health Study. Stroke 1994;25:318-27.

9 Ylikoski A, Erkinjuntti T, Raininko R, et al. White matter hyperintensities on MRI in the neurological non diseased elderly. Analysis of cohorts of consecutive subjects aged 55 to 85 years living at home. Stroke 1995:26:1171-7.

10 Day JJ, Freer CE, Dixon AK, et al. Magnetic resonance imaging of the brain and brain stem in elderly patients with dizziness. Age Ageing 1990; 19:144-50

11 Kerber KA, Enrietto JA, Jacobson KM, et al. Disequilibrium in older people: a prospective study. Neurology 1998;51:574-80.

12 Tell GS, Lefkowitz DS, Diehr P, et al. Relationship between balance and abnormalities in cerebral magnetic resonance imaging in older adults. Arch Neurol 1998;55:73-9.

13 Bogousslavsky J, Maeder P, Regli F, et al. Pure midbrain infarction: clinical syndromes, MRI and etiologic patterns. Neurology 1994;44:2032-40 